\title{
Medidas de desfechos clínicos na meniscectomia artroscópica: Pontuações de joelho segundo médicos e pacientes
}

\section{Clinical Outcome Measures in Arthroscopic Meniscectomy: Clinician versus Patient Completed Knee Scores}

\author{
Oday Al-Dadah ${ }^{1,20} \quad$ Lee Shepstone ${ }^{3} \quad$ Simon T. Donell ${ }^{3}$
}

${ }^{1}$ Translational and Clinical Research Institute, Faculty of Medical Sciences, Newcastle University, Framlington Place, Newcastle-uponTyne, Reino Unido

2 Departamento de Trauma e Cirurgia Ortopédica, South Tyneside Hospital, Harton Lane, South Tyneside, Reino Unido

3 Medical School, University of East Anglia, Earlham Road, Norwich, Reino Unido

Rev Bras Ortop 2022;57(6):1014-1021.
Endereço para correspondência Oday Al-Dadah, MBChB, FRCS (Eng), MD, FRCS (Tr \& Orth), Consultant Orthopaedic Surgeon and Honorary Clinical Senior Lecturer, Newcastle University, Translational and Clinical Research Institute, Framlington Place, Newcastle-upon-Tyne, Tyne and Wear NE2 4HH, United Kingdom (e-mail: odayaldadah@hotmail.com).

\section{Resumo}

\section{Palavras-chave}

- menisco

- meniscectomia

- medidas de resultados relatados pelo paciente

- escore de Lysholm para joelho
Objetivo O objetivo do presente estudo foi investigar a diferença entre instrumentos de desfechos preenchidos por médicos e pacientes na detecção de melhora após a meniscectomia artroscópica para tratamento de rupturas de menisco.

Métodos Trinta e quatro pacientes com rupturas de menisco foram avaliados de forma prospectiva usando 9 medidas de desfechos clínicos. Os cinco instrumentos de avaliação de joelho respondidos por médicos foram o Escore de Atividade de Tegner, o Escore de Joelho de Lysholm, o Escore de Joelho de Cincinnati, o Escore de Exame do Joelho do International Knee Documentation Committee (IKDC, na sigla em inglês) e o Escore de Classificação do Menisco de Tapper e Hoover. Os quatro instrumentos de avaliação do joelho respondidos por pacientes foram o Escore Subjetivo do Joelho do IKDC, a Pesquisa de Desfecho de Joelho - Escala de Atividades de Vida Diária (KOSADLS, na sigla em inglês), o Formulário Curto de Pesquisa em Saúde de 12 Itens (SF-12, na sigla em inglês) e o Escore de Desfecho de Osteoartrite e Lesões no Joelho (KOOS, na sigla em inglês). Vinte e nove dos 34 pacientes foram submetidos a uma meniscectomia artroscópica e reavaliados com todos os 9 instrumentos na sua consulta de acompanhamento. recebido

05 de Abril de 2021

aceito

13 de Agosto de 2021

Publicado on-line

de Janeiro 21, 2022
DOI https://doi.org/

$10.1055 / \mathrm{s}-0041-1740470$. ISSN $0102-3616$ (c) 2022. Sociedade Brasileira de Ortopedia e Traumatologia. All rights reserved.

This is an open access article published by Thieme under the terms of the Creative Commons Attribution-NonDerivative-NonCommercial-License, permitting copying and reproduction so long as the original work is given appropriate credit. Contents may not be used for commercial purposes, or adapted, remixed, transformed or built upon. (https://creativecommons.org/ licenses/by-nc-nd/4.0/)

Thieme Revinter Publicações Ltda., Rua do Matoso 170, Rio de Janeiro, RJ, CEP 20270-135, Brazil 
Resultados Uma melhora longitudinal significativa foi observada em 4 dos 5 instrumentos respondidos por médicos (Tegner $[p<0,001]$, Lysholm $[p=0,004]$, Cincinnati $[p=0,002]$ e Tapper e Hoover $[p<0,001]$, mas não no IKDC $[p=0,332])$. Por outro lado, o Escore Subjetivo do Joelho do $\operatorname{IKDC}(p=0,021)$ foi o único instrumento respondido por pacientes a demonstrar melhora pós-operatória significativa.

Conclusão De modo geral, os instrumentos respondidos por médicos foram considerados inconsistentes em relação àqueles respondidos por pacientes. $\mathrm{O}$ modo de administração dos instrumentos pode ter influência significativa nos resultados, tanto para fins de pesquisa quanto para a prática clínica. A combinação de um instrumento respondido pelo médico com um instrumento respondido pelo paciente pode ser uma abordagem mais equilibrada para a avaliação e a quantificação das rupturas do menisco e do desfecho após a meniscectomia artroscópica.

\section{Abstract}

\section{Keywords}

- meniscus

- meniscectomy

- patient reported outcome measures

- Lysholm knee score
Objective The aim of the present study was to investigate the difference between clinician-completed and patient-completed outcome scores in detecting improvement following arthroscopic meniscectomy in patients with meniscal tears of the knee.

Methods Thirty-four patients with meniscal tears were prospectively assessed using 9 clinical outcome measures. The five clinician-completed knee scores included the Tegner Activity Score, the Lysholm Knee Score, the Cincinnati Knee Score, the International Knee Documentation Committee (IKDC) Examination Knee Score, and the Tapper and Hoover Meniscal Grading Score. The four patient-completed knee scores included the IKDC Subjective Knee Score, the Knee Outcome Survey - Activities of Daily Living Scale (KOS-ADLS), the Short Form-12 Item Health Survey (SF-12), and the Knee Injury and Osteoarthritis Outcome Score (KOOS). Twenty-nine of the 34 patients underwent an arthroscopic meniscectomy and were reassessed with all 9 outcome scores upon their follow-up review.

Results A significant longitudinal improvement was observed in 4 of the 5 cliniciancompleted scores (Tegner $[p<0.001]$, Lysholm $[p=0.004]$, Cincinnati $[p=0.002]$ and Tapper and Hoover $[p<0.001]$, but not in the IKDC Examination [ $p=0.332]$. However, the IKDC Subjective score $(p=0.021)$ was the only patient-completed score to demonstrate significant improvement postoperatively.

Conclusion Overall, clinician-completed scoring systems were found to be inconsistent with those of patient-completed instruments. The mode of administering outcome measures can have a significant influence on the outcome results both for research and for clinical practice. A combination of both a clinician-completed with a patient-completed instrument may be a more balanced approach to assessing and quantifying meniscus tears and the outcome following arthroscopic meniscectomy.

\section{Introdução}

As medidas de desfechos clínicos são importantes tanto na pesquisa quanto na prática clínica. Estes instrumentos permitem que clínicos e pesquisadores quantifiquem objetivamente a gravidade da doença ou da lesão ao avaliarem deficiências e incapacidades. Também podem medir proporcionalmente o desfecho da intervenção (ou seja, da cirurgia). A principal força dos instrumentos de escore é a consideração de experiências, preferências e valores dos pacientes. Estes instrumentos podem ajudar a quantificar os desfechos dos serviços clínicos e, assim, trazer boas informações para a formulação de políticas, auxiliando nas difíceis decisões sobre a distribuição de recursos finitos de saúde. Uma das áreas sob escrutínio recente no campo da cirurgia ortopédica eletiva é a eficácia terapêutica da artroscopia do joelho e da meniscectomia em pacientes com rupturas de menisco. ${ }^{1-4}$ Consequentemente, diretrizes nacionais e internacionais produzidas pela British Association for Surgery of the Knee (BASK, na sigla em inglês) ${ }^{5}$ e pela European Society for Sports Traumatology, Knee Surgery and Arthroscopy (ESSKA, na sigla em inglês) ${ }^{6}$ aconselham a cirurgia apenas a pacientes com rupturas de menisco que ainda são sintomáticos após no mínimo 3 meses de tratamento conservador e sem artrite avançada. As conclusões do presente estudo são pertinentes a este debate. Os instrumentos de desfecho têm papel 
importante em todos os campos da medicina. Seus dados são frequentemente coletados em auditorias nacionais, principalmente em especialidades cirúrgicas (como o National Joint Registry do Reino Unido ${ }^{7}$ ).

O instrumento específico para a avaliação de lesões de menisco é o Escore de Classificação do Menisco de Tapper e Hoover $(\mathrm{T} \& H){ }^{8}$ criado em 1969. A princípio, este instrumento era utilizado para a avaliação de pacientes que haviam sido submetidos a meniscectomia. Este instrumento é um sistema de classificação, não um questionário mais tradicional, e é preenchido pelo médico. Ele categoriza o desfecho após a meniscectomia em um de quatro graus (excelente, bom, moderado e ruim). Tanto o escore de joelho de Lysholm quanto a escala de atividade de Tegner demonstraram desempenhos psicométricos aceitáveis na avaliação de pacientes com lesões de menisco. ${ }^{9}$ Mintzer et al. ${ }^{10}$ usaram o escore de Lysholm como instrumento para a avaliação de pacientes com rupturas de menisco submetidos a cirurgia de reparo. O Escore de Desfecho de Osteoartrite e Lesões no Joelho (KOOS, na sigla em inglês) também foi rigorosamente testado em termos de validade, confiabilidade e capacidade de resposta em pacientes com lesões de menisco e sua avaliação após menisectomia parcial artroscópica. ${ }^{11}$

A Pesquisa de Desfecho de Joelho - Escala de Atividades de Vida Diária (KOS-ADLS, na sigla em inglês) tem se mostrado cientificamente robusta na avaliação de pacientes antes e depois da cirurgia de menisco. ${ }^{12}$ Como adjunto ao escore de Lysholm, o Formulário Curto de Pesquisa em Saúde de 36 Itens (SF-36, na sigla em inglês) tem sido usado para a avaliação de pacientes com rupturas de menisco e para a determinação dos desfechos da menisectomia parcial artroscópica ${ }^{13}$ e do reparo de menisco. ${ }^{10}$ Cole et al. ${ }^{14}$ usaram o Formulário Curto de Pesquisa em Saúde de 12 Itens (SF-12, na sigla em inglês), além de vários outros escores específicos de doenças de joelho, para a avaliação dos desfechos do transplante de menisco com aloenxerto em pacientes com sintomas persistentes de dor após meniscectomias anteriores. Koyonos et al. ${ }^{15}$ conduziram um estudo duplo-cego randomizado para investigar a eficácia de injeções intra-articulares de corticosteroides em pacientes com rupturas de menisco que foram submetidos a uma meniscectomia parcial artroscópica e apresentaram alterações osteoartríticas concomitantes no joelho no momento da cirurgia. Estes autores usaram o SF-12, além de vários outros instrumentos de desfecho específicos da doença, para a avaliação dos resultados da sua pesquisa.

O escore de joelho de Cincinnati demonstrou ter excelente validade, confiabilidade e capacidade de resposta na avaliação de pacientes com um amplo espectro de patologias de joelho, inclusive rupturas de menisco. ${ }^{16} \mathrm{O}$ escore subjetivo do joelho do International Knee Documentation Committee (IKDC, na sigla em inglês) demonstrou ser um instrumento válido, confiável e responsivo na avaliação de pacientes com lesões de menisco. ${ }^{17,18}$ Mintzer et al. ${ }^{10}$ e Pujol et al. ${ }^{19}$ usaram o formulário de exame do joelho do IKDC para a avaliação de pacientes com lesões de menisco submetidas a reparo.

A disponibilidade e o uso de diversos instrumentos na avaliação de pacientes com rupturas de menisco têm deficiências. Estudos que utilizaram diversos sistemas de pon- tuação de joelho na avaliação de pacientes com lesão de menisco ${ }^{14,15}$ revelaram a disparidade de resultados obtidos com os diferentes instrumentos. Isto enfatiza ainda mais a falta de padronização observada entre os instrumentos hoje utilizados. Assim, os pesquisadores rotineiramente usam dois ou mais instrumentos de desfechos clínicos para a análise dos resultados das suas intervenções. A comparação de resultados de estudos que investigaram a mesma área, mas usaram instrumentos diferentes, é repleta de limitações e dificuldades.

Os instrumentos de desfechos clínicos podem ser amplamente categorizados entre aqueles respondidos por médicos ou por pacientes. $O$ objetivo do presente estudo foi comparar os resultados de escores de joelho respondidos pelo médico e pelo paciente após a meniscectomia parcial artroscópica para avaliar se há uma diferença entre os dois métodos de administração dos instrumentos.

\section{Materiais e Métodos}

O presente estudo foi integralmente aprovado pelo Comitê de Ética em Pesquisa e pelo Comitê de Governança em Pesquisa. Todos os pacientes assinaram o termo de consentimento livre e esclarecido para participação. O presente estudo terapêutico é um estudo de coorte longitudinal prospectivo cujos dados fizeram parte da tese de doutorado do primeiro autor. Um estudo semelhante, com coorte inteiramente distinta, formada por pacientes com ruptura do ligamento cruzado anterior, já foi publicado. ${ }^{20}$ Alguns dados deste estudo também foram utilizados no braço terapêutico de outro estudo de caso-controle submetido para publicação.

No total, 50 pacientes foram recrutados para o estudo (com base no cálculo do tamanho da amostra da pesquisa mais ampla da qual esses dados fazem parte). A - Tabela 1 mostra os dados demográficos de todos os participantes. Os pacientes incluídos tinham entre 16 e 45 anos de idade. Os critérios de exclusão foram procedimentos de reparo de menisco, lesão concomitante nos ligamentos do joelho, lesões importantes da cartilagem articular do joelho, histórico significativo de patologia do tornozelo ou do quadril, sintomas relacionados à coluna lombar (inclusive radiculopatia em qualquer um dos membros), doença neurológica ou vestibular, diabetes, uso regular de analgésicos opioides ou

Tabela 1 Dados demográficos dos pacientes

\begin{tabular}{|l|l|}
\hline & $\begin{array}{l}\text { Pacientes do } \\
\text { grupo Menisco } \\
(\boldsymbol{n}=50)\end{array}$ \\
\hline Idade média (anos) (DP) & $34(9)$ \\
\hline Homens:Mulheres & $37: 13$ \\
\hline Joelho acometido (direito:esquerdo) & $29: 21$ \\
\hline Altura média (m) (DP) & $1,74(0,1)$ \\
\hline Peso médio (kg) (DP) & $83,9(18,6)$ \\
\hline IMC médio $\left(\mathrm{kg} / \mathrm{m}^{2}\right)(\mathrm{DP})$ & $27,6(4,9)$ \\
\hline
\end{tabular}

Abreviaturas: DP, desvio padrão; IMC, índice de massa corporal. 


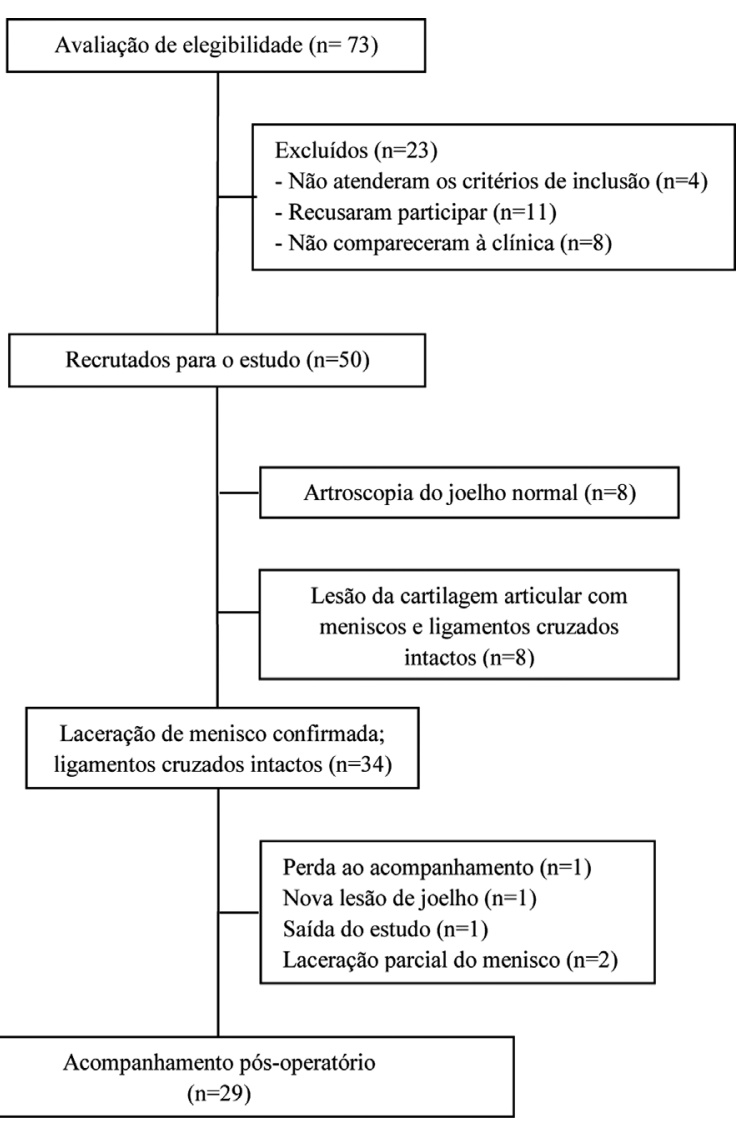

Fig. 1 Fluxo de pacientes do estudo.

implante de dispositivos incompatíveis com a ressonância magnética (RM).

O tempo médio entre a lesão e a revisão clínica foi de 63 semanas (desvio padrão $[\mathrm{DP}]=41$ ). 0 diagnóstico de ruptura isolada de menisco, com cartilagem e ligamentos íntegros, foi estabelecido por exame clínico e RM do joelho acometido. Estes achados foram confirmados na artroscopia do joelho em todos os pacientes. A anamnese e o exame físico confirmaram a normalidade do joelho contralateral. A - Figura 1 ilustra o fluxo de pacientes no grupo Menisco ao longo do estudo e a - Tabela 2 mostra os mecanismos de lesão. Dos 34 pacientes com rupturas de menisco, 16 apresentavam ruptura medial, 17 tinham ruptura lateral, e 1 paciente apresentava lesões medial e lateral simultâneas. Trinta e dois destes pacientes foram submetidos a uma meniscectomia parcial artroscópica. Dois pacientes tinham rupturas parciais do menisco. Um apresentava ruptura parcial da

Tabela 2 Mecanismo da lesão $(n=50)$

\begin{tabular}{|l|l|l|l|}
\hline Causa da lesão & $\boldsymbol{n}(\%)$ & Causa da lesão & $\boldsymbol{n}(\%)$ \\
\hline $\begin{array}{l}\text { Sintomas de } \\
\text { aparecimento } \\
\text { espontâneo }\end{array}$ & $22(44)$ & Futebol & $5(10)$ \\
\hline Queda & $10(20)$ & Corrida & $5(10)$ \\
\hline Traumatismo menor & $6(12)$ & $\begin{array}{l}\text { Acidente } \\
\text { de trânsito }\end{array}$ & $2(4)$ \\
\hline
\end{tabular}

superfície superior do corno posterior do menisco medial e o outro tinha uma ruptura semelhante, mas do menisco lateral. Nenhum destes dois pacientes foi submetido à meniscectomia. Oito pacientes apresentaram lesões significativas na cartilagem articular (grau III/IV na escala modificada de Outerbridge $)^{21-25}$ do compartimento tibiofemoral medial ou lateral ou no compartimento patelofemoral. 0 tempo médio de acompanhamento foi de 13,4 semanas $(\mathrm{DP}=3,8)$ após a cirurgia.

No total, nove instrumentos de desfechos clínicos foram utilizados no presente estudo. Cinco eram instrumentos preenchidos pelo médico e quatro eram instrumentos preenchidos pelo paciente. Estes escores de joelho foram escolhidos por serem os mais usados na literatura, à exceção do Escore de T\&H, que foi incluído por ser o único instrumento desenvolvido especificamente para a avaliação de lesões de menisco. Todos os instrumentos de desfechos clínicos foram validados para o uso na avaliação de pacientes com lesões no joelho. Os escores de joelho preenchidos por médicos foram aplicados no momento do comparecimento dos pacientes à clínica de pesquisa. Os escores respondidos por pacientes foram enviados para os participantes $\sim 7$ dias antes do seu comparecimento à clínica de pesquisa. Portanto, os participantes preencheram os instrumentos em seu próprio tempo e suas avaliação e percepção não foram influenciadas pelo comprometimento funcional do joelho. Todos os indivíduos foram avaliados com estes instrumentos no início do estudo (antes da cirurgia) e no período pós-operatório (pacientes acompanhados após a cirurgia).

\section{Escores de Joelho Preenchidos pelo Médico}

Os instrumentos preenchidos pelo médico foram:

- Escala de Atividade de Tegner ${ }^{26}$

- Escore de Joelho de Lysholm ${ }^{26}$

- Escore de Joelho de Cincinnati ${ }^{27-29}$

- Escore de Exame do Joelho do IKDC ${ }^{30,31}$

- Escore de Classificação do Menisco de Tapper e Hoover ${ }^{8}$ $(\mathrm{T} \& \mathrm{H})$

\section{Escores de Joelho Preenchidos pelo Paciente}

Os instrumentos preenchidos pelo paciente foram:

- Escore Subjetivo do Joelho do IKDC ${ }^{18,32}$

- Pesquisa de Desfecho de Joelho - Escala de Atividades de Vida Diária ${ }^{12}$ (KOS-ADLS)

- Formulário Curto de Pesquisa em Saúde de 12 Itens $^{33}$ (SF-12)

- Escore de Desfecho de Osteoartrite e Lesões no Joelho 34,35 (KOOS)

\section{Análise Estatística}

Todas as variáveis de dados contínuos exibiram distribuição normal em ambos os histogramas plotados e no teste de Shapiro-Wilk. Os resultados foram avaliados pelo teste t de Student pareado para análises dentro do grupo de variáveis contínuas; os resultados dos dois instrumentos do IKDC e do escore T\&H eram variáveis ordinais categóricas e foram 
Tabela 3 Comparação dos escores de desfecho do joelho antes $(n=34)$ e depois $(n=29)$ da cirurgia

\begin{tabular}{|l|l|l|l|l|l|}
\hline & Antes da cirurgia & Depois da cirurgia & valor- $\boldsymbol{p}^{\mathbf{1}}$ & \multirow{2}{*}{ 95\% Cl } & \multirow{2}{*}{ Diferença média } \\
\cline { 2 - 5 } & Média (DP) & Média (DP) & & & \multicolumn{2}{l}{1.3} \\
\hline Tegner & $3.1(1.2)$ & $4.4(1.3)$ & $0.001^{*}$ & 0.77 para 1.82 & 13.6 \\
\hline Lysholm & $72.6(21.7)$ & $86.2(12.2)$ & $0.004^{*}$ & 4.72 para 22.40 & 16.8 \\
\hline IKDC (Subjetivo) & $66.7(23.9)$ & $83.5(16.4)$ & $0.002^{*}$ & 6.82 para 26.74 & 11.8 \\
\hline KOS-ADLS & $53.2(19.3)$ & $65.0(21.0)$ & $0.021^{*}$ & 1.93 para 21.65 & 5.7 \\
\hline SF-12 PCS & $70.9(18.5)$ & $76.6(17.8)$ & 0.177 & -2.73 para 14.10 & 3.7 \\
\hline SF-12 MCS & $41.6(10.9)$ & $45.3(9.1)$ & 0.138 & -1.26 para 8.63 & 0.5 \\
\hline KOOS & $51.7(11.4)$ & $52.2(11.8)$ & 0.719 & -2.47 para 3.52 & 0.5 \\
\hline Sintomas & & & & & 8.2 \\
\hline Dor & $65.6(16.7)$ & $73.7(19.5)$ & 0.050 & 0.01 para 16.33 & 8.6 \\
\hline Atividades da vida diária & $75.0(20.7)$ & $83.8(18.5)$ & 0.090 & -1.44 para 18.70 & 8.8 \\
\hline $\begin{array}{l}\text { Esportes e atividades } \\
\text { recreativas }\end{array}$ & $47.9(29.4)$ & $59.1(30.4)$ & 0.092 & -1.97 para 24.47 & 11.3 \\
\hline Qualidade de vida & $37.5(22.0)$ & $51.6(25.6)$ & $0.005^{*}$ & 4.70 para 23.43 & 14.1 \\
\hline
\end{tabular}

Abreviações: DP, desvio padrão; IC, intervalo de confiança; IKDC, International Knee Documentation Committee; KOOS, Escore de Desfecho de Osteoartrite e Lesões no Joelho; KOS-ADLS, Pesquisa de Desfecho de Joelho - Escala de Atividades de Vida Diária; MCS, resumo do componente mental; PCS, resumo do componente físico; SF-12, Formulário Curto de Pesquisa em Saúde de 12 Itens.

*Estatisticamente significativo em $p<0,05$.

${ }^{1}$ Análise do teste $t$ de Student pareado.

analisados pelo teste estatístico não paramétrico apropriado (teste dos postos sinalizados de Wilcoxon). 0 nível de significância estatística foi estabelecido em $p<0,05$. A análise estatística foi realizada no software IBM SPSS Statistics for Windows versão 25.0 (IBM Corp., Armonk, NY, EUA).

\section{Resultados}

A - Tabela 3 mostra os resultados dos instrumentos do grupo Menisco (variáveis contínuas) e a análise estatística longitudinal. Uma melhora longitudinal significativa foi observada nos escores de joelho preenchidos pelos médicos, incluindo Tegner, Lysholm e Cincinnati. Por outro lado, o único instrumento preenchido por pacientes a apresentar melhora longitudinal significativa no período pós-operatório foi o escore subjetivo do IKDC. A - Tabela 4 mostra que houve uma diferença significativa entre o escore médio de Tegner no período pré-operatório em comparação com o escore anterior à lesão. Embora tenha havido uma melhora significativa no escore de Tegner após a cirurgia (- Tabela 3), os pacientes do grupo Menisco ainda não haviam retornado ao seu nível de atividade anterior à lesão no momento do acompanhamento, com manutenção de uma diferença significativa. As - Figuras 2 e $\mathbf{3}$ mostram a análise estatística longitudinal dos instrumentos do IKDC e T\&H (respectivamente). Ambos são instrumentos preenchidos por médicos. Houve uma melhora significativa no escore T\&H após a meniscectomia. No entanto, isto não ocorreu com o instrumento do IKDC.
Tabela 4 Comparação dos escores de atividade de Tegner antes $(n=34)$ e depois $(n=29)$ da cirurgia com o escore anterior à lesão (média $=5,7$; desvio padrão $=1,3$ )

\begin{tabular}{|l|l|l|l|}
\hline & valor-p $^{1}$ & IC95\% & $\begin{array}{l}\text { Diferença } \\
\text { média }\end{array}$ \\
\hline $\begin{array}{l}\text { Tegner antes } \\
\text { da lesão } \\
\text { versus antes } \\
\text { da cirurgia }\end{array}$ & $<0,001^{*}$ & 2,0 para 3,29 & 2,6 \\
\hline $\begin{array}{l}\text { Tegner antes } \\
\text { da lesão } \\
\text { versus depois } \\
\text { da cirurgia }\end{array}$ & $0,001^{*}$ & 0,68 para 2,32 & 1,3 \\
\hline
\end{tabular}

Abreviação: IC, intervalo de confiança.

${ }^{*}$ Estatisticamente significativo em $p<0,05$.

${ }^{1}$ Análise do teste $t$ de Student pareado.

\section{Discussão}

Os resultados do presente estudo revelaram uma melhora significativa em quatro dos cinco instrumentos preenchidos por médicos (à exceção do escore de exame do IKDC) após a meniscectomia parcial artroscópica. No entanto, o escore subjetivo do IKDC foi o único instrumento preenchido por pacientes a demonstrar uma melhora longitudinal significativa.

Um achado interessante foi a melhora estatisticamente significativa do escore subjetivo do IKDC, mas a ausência de melhora significativa no escore de exame do IKDC. Uma 


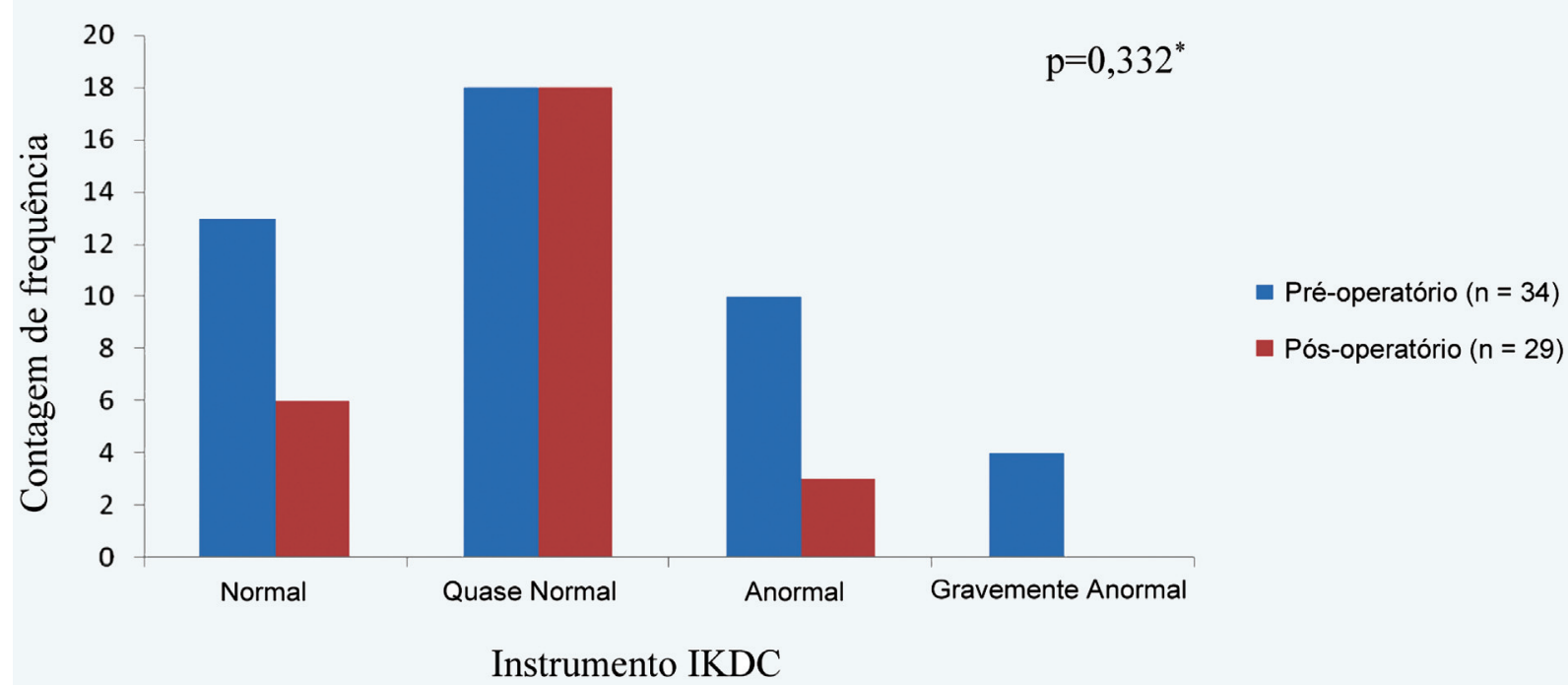

Fig. 2 Comparação dos escores de exame do joelho do International Knee Documentation Committee (IKDC) (contagens de frequência) dos pacientes do grupo Menisco antes e depois da meniscectomia. *Análise dos postos sinalizados de Wilcoxon.

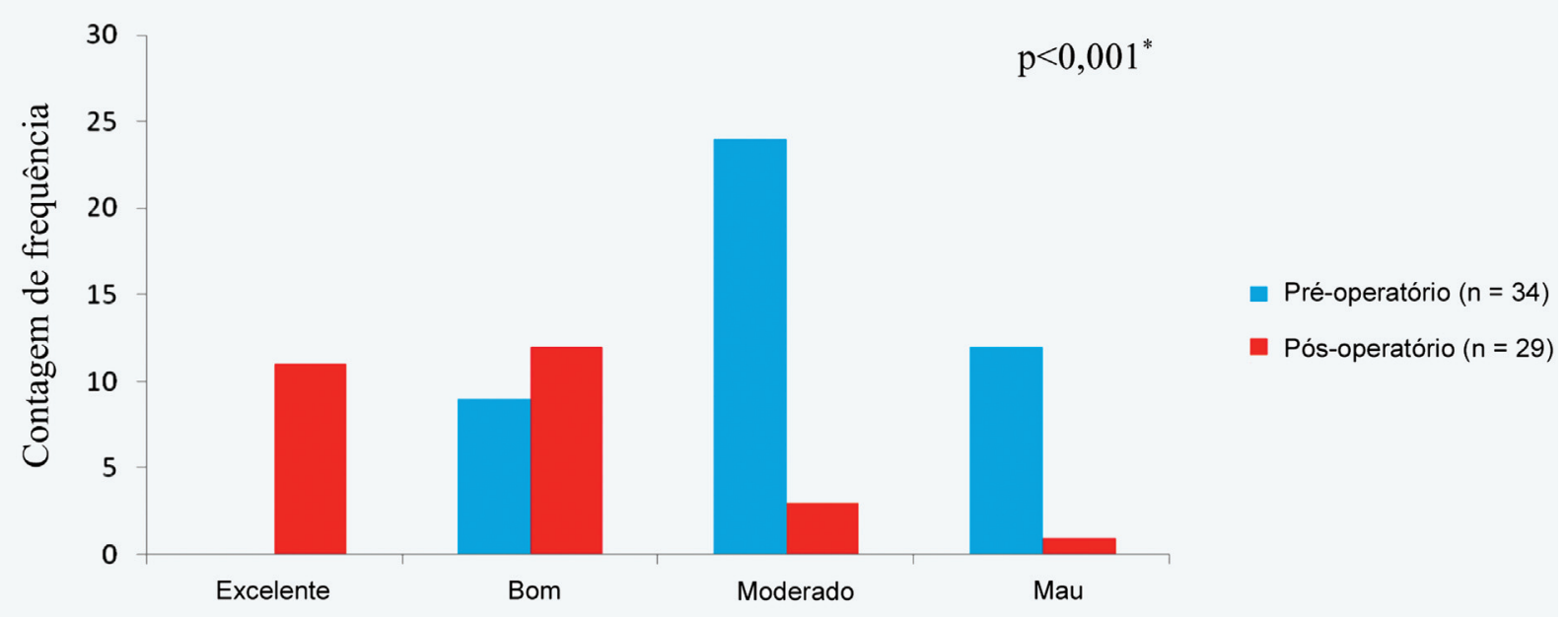

Instrumento de Tapper e Hoover

Fig. 3 Comparação dos escores de classificação do menisco de Tapper e Hoover (contagens de frequência) dos pacientes do grupo Menisco antes e depois da meniscectomia. *Análise dos postos sinalizados de Wilcoxon.

melhora significativa no instrumento T\&H também foi observada, em contraste com o escore de exame do IKDC, que é mais elaborado. Isto pode ser explicado pelo fato de que 0 escore de exame do IKDC foi originalmente desenvolvido para a avaliação de lesões ligamentares do joelho com grande ênfase no teste de frouxidão ligamentar, ${ }^{31}$ e não nos sintomas relacionados às rupturas de menisco, como o escore T\&H. ${ }^{8}$ O escore subjetivo do IKDC $^{17,18}$ foi projetado para abranger uma gama maior de patologias do joelho do que $o$ instrumento de exame do IKDC, o que poderia explicar por que a pontuação anterior foi considerada mais responsiva neste respeito. Os únicos dois subgrupos estatisticamente significativos do escore KOOS foram atividades de vida diária $(p=0,042)$ e qualidade de vida $(p=0,005)$. O primeiro resultado teve significância estatística limítrofe e pode represen- tar um erro estatístico do tipo I à luz dos demais achados do subgrupo KOOS. O segundo resultado pode ser um reflexo de modificações do estilo de vida ou simplesmente da aceitação dos sintomas pelos pacientes e da sua maior perseverança nas atividades diárias normais.

De maneira geral, há uma inconsistência entre as medidas de desfecho clínico segundo médicos e pacientes na avaliação de indivíduos com lesões de menisco após a meniscectomia parcial. A discordância entre estas duas técnicas de determinação de desfechos pode ser explicada pelo possível viés do entrevistador, o qual pode levar o cirurgião a subestimar inconscientemente quaisquer respostas desfavoráveis do paciente. Da mesma forma, os próprios pacientes podem estar menos inclinados a admitir uma resposta desfavorável em uma consulta presencial. Os instrumentos preenchidos 
por pacientes podem, portanto, dar uma indicação melhor do grau de comprometimento funcional do joelho após a lesão de menisco e a cirurgia. Uma melhora significativa foi observada no escore de atividade de Tegner ao comparar os achados pré-operatórios com os pós-operatórios. No entanto, também existiu uma diferença significativa ao comparar os resultados pós-operatórios com aqueles anteriores à lesão, implicando que os pacientes no grupo Menisco ainda não haviam se recuperado por completo e retornado ao nível original de atividade 3 meses após a meniscectomia parcial artroscópica. Este achado foi surpreendente, uma vez que os pacientes do grupo Menisco não foram submetidos ao mesmo programa de reabilitação rigoroso e às restrições de atividades instituídos após a cirurgia de reconstrução do ligamento cruzado anterior. O período de acompanhamento de 3 meses pode ser responsável por este resultado e pode ter influenciado a capacidade limitada de resposta dos escores de joelho preenchidos por pacientes após a intervenção cirúrgica. Portanto, um resultado estatisticamente significativo na análise longitudinal das medidas de desfecho segundo os pacientes do grupo Menisco poderia ser observado caso o período de acompanhamento fosse maior. No entanto, houve uma melhora significativa nos instrumentos preenchidos por médicos no mesmo período de acompanhamento. Outro fator do paciente que pode influenciar os resultados é a possível compreensão limitada das perguntas feitas e, portanto, da capacidade de preencher adequadamente os instrumentos.

A forma de administração de um instrumento de avaliação do joelho no ambiente clínico ou de pesquisa é, portanto, importante, pois pode ter influência significativa nos resultados. Consequentemente, o uso combinado de um instrumento de desfecho clínico preenchido pelo médico e um instrumento de desfecho clínico preenchido pelo paciente pode ser o melhor formato de avaliação em estudos relacionados a lesões de menisco. Com base nos resultados do presente estudo, o instrumento preenchido por médicos recomendado para a prática clínica é o $\mathrm{T} \& H$, devido à sua facilidade de uso e capacidade de resposta após a meniscectomia parcial. Da mesma forma, o instrumento preenchido por pacientes recomendado é o escore subjetivo do joelho do IKDC, pois foi o único dentre os sistemas respondidos pelos pacientes a ser considerado responsivo após a cirurgia de menisco.

Dentre as limitações do presente estudo estão a ausência de avaliação radiológica do alinhamento geral dos membros inferiores, a qual poderia ter auxiliado na detecção da influência de quaisquer deformidades em varo ou valgo nos escores no contexto de rupturas de menisco; tal avaliação pode ser considerada por futuros pesquisadores. Além disso, a inclusão de um número maior de pacientes permitiria uma comparação significativa de subgrupos de dados relativos a rupturas mediais e laterais de menisco.

\section{Conclusão}

De modo geral, os resultados longitudinais dos instrumentos de desfecho preenchidos por médicos foram considerados inconsistentes com aqueles preenchidos por pacientes. Uma combinação de um instrumento preenchido pelo médico com um instrumento preenchido pelo paciente pode ser uma abordagem mais equilibrada para a avaliação e a quantificação das rupturas de menisco e do desfecho após a meniscectomia artroscópica.

\section{Financiamento}

O presente estudo foi financiado pelo Gwen Fish Charity Trust, pelo Norfolk and Norwich University Hospital Research Fund e pelo Action Arthritis Charity Trust.

Conflito de Interesses

Os autores declaram não haver conflito de interesses.

\section{Referências}

1 Kise NJ, Risberg MA, Stensrud S, Ranstam J, Engebretsen L, Roos EM. Exercise therapy versus arthroscopic partial meniscectomy for degenerative meniscal tear in middle aged patients: randomised controlled trial with two year follow-up. BMJ 2016;354: i3740

2 Sihvonen R, Paavola M, Malmivaara A, et al. Arthroscopic partial meniscectomy for a degenerative meniscus tear: a 5 year followup of the placebo-surgery controlled FIDELITY (Finnish Degenerative Meniscus Lesion Study) trial. Br J Sports Med 2020;54(22): 1332-1339

3 Sihvonen R, Paavola M, Malmivaara A, et al. Arthroscopic partial meniscectomy versus sham surgery for a degenerative meniscal tear. N Engl J Med 2013;369(26):2515-2524

4 Thorlund JB, Juhl CB, Roos EM, Lohmander LS. Arthroscopic surgery for degenerative knee: systematic review and metaanalysis of benefits and harms. BMJ 2015;350:h2747

5 Abram SGF, Beard DJ Price AJBASK Meniscal Working Group. Arthroscopic meniscal surgery: a national society treatment guideline and consensus statement. Bone Joint J 2019;101-B (06):652-659

6 Beaufils P, Becker R, Kopf S, et al. Surgical management of degenerative meniscus lesions: the 2016 ESSKA meniscus consensus. Knee Surg Sports Traumatol Arthrosc 2017;25(02):335-346

7 The National Joint Registry - Patient Reported Outcome Measures Available from: https://www.njrcentre.org.uk/njrcentre/Research/ NJR-PROMs

8 Tapper EM, Hoover NW. Late results after meniscectomy. J Bone Joint Surg Am 1969;51(03):517-526

9 Briggs KK, Kocher MS, Rodkey WG, Steadman JR. Reliability, validity, and responsiveness of the Lysholm knee score and Tegner activity scale for patients with meniscal injury of the knee. J Bone Joint Surg Am 2006;88(04):698-705

10 Mintzer CM, Richmond JC, Taylor J. Meniscal repair in the young athlete. Am J Sports Med 1998;26(05):630-633

11 Roos EM, Roos HP, Ekdahl C, Lohmander LS. Knee injury and Osteoarthritis Outcome Score (KOOS)-validation of a Swedish version. Scand J Med Sci Sports 1998;8(06):439-448

12 Irrgang JJ, Snyder-Mackler L, Wainner RS, Fu FH, Harner CD. Development of a patient-reported measure of function of the knee. J Bone Joint Surg Am 1998;80(08):1132-1145

13 Katz JN, Harris TM, Larson MG, et al. Predictors of functional outcomes after arthroscopic partial meniscectomy. J Rheumatol 1992;19(12):1938-1942

14 Cole BJ, Dennis MG, Lee SJ, et al. Prospective evaluation of allograft meniscus transplantation: a minimum 2-year follow-up. Am J Sports Med 2006;34(06):919-927

15 Koyonos L, Yanke AB, McNickle AG, et al. A randomized, prospective, double-blind study to investigate the effectiveness of adding 
DepoMedrol to a local anesthetic injection in postmeniscectomy patients with osteoarthritis of the knee. Am J Sports Med 2009;37 (06):1077-1082

16 Marx RG, Jones EC, Allen AA, et al. Reliability, validity, and responsiveness of four knee outcome scales for athletic patients. J Bone Joint Surg Am 2001;83(10):1459-1469

17 Anderson AF, Irrgang JJ, Kocher MS, Mann BJ, Harrast JJInternational Knee Documentation Committee. The International Knee Documentation Committee Subjective Knee Evaluation Form: normative data. Am J Sports Med 2006;34(01):128-135

18 Irrgang JJ, Anderson AF, Boland AL, et al. Development and validation of the international knee documentation committee subjective knee form. Am J Sports Med 2001;29(05): 600-613

19 Pujol N, Panarella L, Selmi TA, Neyret P, Fithian D, Beaufils P. Meniscal healing after meniscal repair: a CT arthrography assessment. Am J Sports Med 2008;36(08):1489-1495

20 Al-Dadah O, Shepstone L, Donell ST. Clinical outcome measures in anterior cruciate ligament reconstruction: Clinician vs patient completed knee scores. Surgeon 2021;19(06):e353-e360

21 Cameron ML, Briggs KK, Steadman JR. Reproducibility and reliability of the outerbridge classification for grading chondral lesions of the knee arthroscopically. Am J Sports Med 2003;31 (01):83-86

22 Curl WW, Krome J, Gordon ES, Rushing J, Smith BP, Poehling GG. Cartilage injuries: a review of 31,516 knee arthroscopies. Arthroscopy 1997;13(04):456-460

23 Outerbridge RE. The etiology of chondromalacia patellae. J Bone Joint Surg Br 1961;43-B:752-757

24 Outerbridge RE, Dunlop JA. The problem of chondromalacia patellae. Clin Orthop Relat Res 1975;(110):177-196

25 Spindler KP, Warren TA, Callison JC Jr, Secic M, Fleisch SB, Wright RW. Clinical outcome at a minimum of five years after recons- truction of the anterior cruciate ligament. J Bone Joint Surg Am 2005;87(08):1673-1679

26 Tegner Y, Lysholm J. Rating systems in the evaluation of knee ligament injuries. Clin Orthop Relat Res 1985;(198):43-49

27 Bentley G, Biant LC, Carrington RW, et al. A prospective, randomised comparison of autologous chondrocyte implantation versus mosaicplasty for osteochondral defects in the knee. J Bone Joint Surg Br 2003;85(02):223-230

28 Noyes FR, Barber SD, Mangine RE. Bone-patellar ligament-bone and fascia lata allografts for reconstruction of the anterior cruciate ligament. J Bone Joint Surg Am 1990;72(08):1125-1136

29 Noyes FR, Barber SD, Mooar LA. A rationale for assessing sports activity levels and limitations in knee disorders. Clin Orthop Relat Res 1989;(246):238-249

30 Forms IKDC. The American Orthopaedic Society for Sports Medicine. 2000 Available from: <http://www.sportsmed. org/tabs/research/ikdc.aspx >

31 Hefti F, Müller W, Jakob RP, Stäubli HU. Evaluation of knee ligament injuries with the IKDC form. Knee Surg Sports Traumatol Arthrosc 1993;1(3-4):226-234

32 Irrgang JJ, Anderson AF. Development and validation of healthrelated quality of life measures for the knee. Clin Orthop Relat Res 2002;(402):95-109

33 Ware J Jr, Kosinski M, Keller SDA. A 12-Item Short-Form Health Survey: construction of scales and preliminary tests of reliability and validity. Med Care 1996;34(03):220-233

34 Roos EM, Lohmander LS. The Knee injury and Osteoarthritis Outcome Score (KOOS): from joint injury to osteoarthritis. Health Qual Life Outcomes 2003;1:64

35 Roos EM, Roos HP, Lohmander LS, Ekdahl C, Beynnon BD. Knee Injury and Osteoarthritis Outcome Score (KOOS)-development of a self-administered outcome measure. J Orthop Sports Phys Ther 1998;28(02):88-96 